\title{
Partial Integrated Guidance and Control Design for Hypersonic Vehicle in Dive Phase
}

\author{
Q.K. Liu ${ }^{a, \star}$, J. Chen ${ }^{b}$, L.X. Wang ${ }^{c}$ \\ Xi'an Research Institute of High-Tech, Xi'an, China \\ alqk_rkue@foxmail.com, bchenjian_rk@126.com, ${ }^{\mathrm{c}}$ wanglixin@126.com
}

\begin{abstract}
Keywords: Hypersonic vehicle, dive phase, terminal sliding mode control, partial integrated guidance and control
\end{abstract}

\begin{abstract}
Considering the potential unstable problem using Euler angle, the quaternion is used to take its place in proposed method. What is more, A partial integrated guidance control (PIGC) model is derived based on six-degree-freedom model to solve the guidance and control problems of hypersonic vehicle in dive phase, the desired body rates is calculated in the outer loop as virtual control signals by making use of the information of the state of vehicle and the relative motion between vehicle and target, and the fin deflection signals is obtained in inner loop by using the sliding mode controller to track the body rate commands. And the results of the simulation demonstrate the effectiveness of the proposed method.
\end{abstract}

\section{Introduction}

Hypersonic vehicles have drawn various attention due to its high speed and maneuverability, and have high value in military affair and civil area [1]. However, because of the complex aerodynamic shape, high speed, and complicated flight environment, the aerodynamic and motion models of hypersonic vehicles are highly nonlinear and rapidly vary in time, exhibit severe coupling and contain various uncertainties. As the result, designing a guidance and control system may face great challenges [2].

The dive phase is the last phase for hypersonic vehicles, so it is of great importance for whole mission. The existing literature has done a lot of research on the guidance and control problem, and applied so many theory on it. For example, optimal control [3], sliding mode control [4], backstepping [5] ,dynamic inversion [6], nonlinear control methods, as well as Fuzzy control ${ }^{[7]}$, predictive control ${ }^{[8]}$ and neural network ${ }^{[9]}$ and other modern control methods. But most of them only focus on one side of the guidance and control problem, this article tries to solve them together.

Traditional guidance and control system designing for missiles is always based on separation theory ${ }^{[10]}$, which means that the coupling between the guidance and control loop is neglected, and the design of the two circuits is carried out independently, so the guidance loop doesn't include the information of attitudes and body rates. When the missiles and target have high relative velocity, the guidance command may exceed the constraint of the control loop. At the same time, the control loop also exclude the information of the relative distance and speed between missiles and target, which leads to it unable to adjust the response speed according to the relative distance of the target.

This structure can't adapt to the situation when the relative velocity between vehicle and target is high. Integrated guidance and control only use single loop to realize the guidance and control system, of course. it simplifies the structure and makes full use of the information of vehicle. However this method fails to take the inherit separation between translational and rotational dynamics into account, which brings the difficulty in application. IGC formulations ignore this fundamental feature by being overambitious to generate the control surface deflections directly. Because of this, the tuning of design parameters also becomes extremely difficult in IGC and quite often becomes engagement specific as well, which is strongly undesirable. This is because the tuning process must be done in a blind manner in such a way that it must lead to the generation of sufficient moments to turn the vehicle toward the target without destabilizing the body rates. The preceding difficulty can be solved by 
retaining one the good features of the IGC framework. Note that to retain the philosophy and advantages of IGC, this should be done by manipulating the 6DOF model directly, and there should not be any lateral acceleration generation loop via an equivalent kinematic or point mass dynamics. In fact, the proposed PIGC design concept does exactly that and hence, one can interpret it as an attempt to combine the best of the two methods.A 6DOF PIGC scheme of the hypersonic vehicle in the dive phase is proposed. The 6DOF dynamic and motion models are established, and a two-loop controlled structure is applied in the deduction of PIGC algorithm: the outer loop get the desire body rates signal, and the inner loop get the command fin deflection commands by tacking the signals from outer loop.

\section{Mathematical models}

\subsection{Six DOF Model of Hypersonic Vehicle}

The 6DOF dynamic model in the body coordinate system is given as follows ${ }^{[12,13]}$ :

$$
\left\{\begin{array}{l}
\dot{u}=\omega_{z} v-\omega_{y} w+F_{x} / m-t_{12} g \\
\dot{v}=\omega_{x} w-\omega_{z} u+F_{y} / m-t_{22} g \\
\dot{w}=\omega_{y} u-\omega_{x} v+F_{z} / m-t_{32} g \\
\dot{\omega}_{x}=\left(q S a C_{l}+\left(I_{y y}-I_{z z}\right) \omega_{z} \omega_{y}\right) / I_{x x} \\
\dot{\omega}_{y}=\left(q S a C_{n}+\left(I_{z z}-I_{x x}\right) \omega_{x} \omega_{z}\right) / I_{y y} \\
\dot{\omega}_{z}=\left(q S c C_{m}+\left(I_{x x}-I_{y y}\right) \omega_{y} \omega_{x}\right) / I_{z z}
\end{array}\right.
$$

Where, $F_{x}=L \sin \alpha-N \cos \alpha \sin \beta-D \cos \alpha \cos \beta, F_{y}=L \cos \alpha+N \sin \alpha \sin \beta+D \sin \alpha \cos \beta$,

$F_{z}=N \cos \beta-D \sin \beta$; $u, v, w$ are the hypersonic vehicle velocity components, $m$ is the mass of the hypersonic vehicle, $g$ is the gravity acceleration, $\alpha, \beta$ are attack angle and slip angle, $D, L, N$ are the aerodynamic drag, lift, and side force, and are given as follow: $D=q S C_{D}, L=q S C_{L}, N=q S C_{N}$.

Where, $q=0.5 \rho V^{2}$ is the dynamic pressure, $\rho$ is the atmospheric density, $V=u^{2}+v^{2}+w^{2}$ is the velocity of the vehicle, $S$ is the aerodynamic reference area $C_{D}, C_{L}, C_{N}, C_{l}, C_{n}$ and $C_{m}$ are the nominal aerodynamic drag, lift, side force coefficients, and the nominal aerodynamic roll, yaw ,pitch moment coefficients respectively. $I_{x x}, I_{y y}$ and $I_{z z}$ are the roll, yaw, and pitch moments of inertia, respectively. $a, c$ are the lateral and longitudinal reference length. All the basic data of the hypersonic vehicle used in this paper can be found in[12]. $\omega_{x}, \omega_{y}$ and $\omega_{z}$ are the body rates components in body frame; $t_{12}, t_{22}, t_{32}$ are the elements of matrix $\boldsymbol{T}_{B I}$, where $\boldsymbol{T}_{B I}$ is the time varying transformation matrix expressed by quaternion $q_{0}, q_{1}, q_{2}, q_{3}$.The quaternions are given by [14]:

$$
\left[\begin{array}{llll}
\dot{q}_{0} & \dot{q}_{1} & \dot{q}_{2} & \dot{q}_{3}
\end{array}\right]^{\mathrm{T}}=\frac{1}{2} \boldsymbol{T}_{1}\left[\begin{array}{lll}
\omega_{x} & \omega_{y} & \omega_{z}
\end{array}\right]^{\mathrm{T}}
$$

Where:

$$
\boldsymbol{T}_{1}=\left[\begin{array}{ccc}
-q_{1} & -q_{2} & -q_{3} \\
q_{0} & -q_{3} & q_{2} \\
q_{3} & q_{0} & -q_{1} \\
-q_{2} & q_{1} & q_{0}
\end{array}\right]
$$

The centroid kinematical equations of the hypersonic vehicle depicted in the ground coordinate system are given below:

$$
\left[\begin{array}{lll}
\dot{x}_{g} & \dot{y}_{g} & \dot{z}_{g}
\end{array}\right]^{\mathrm{T}}=\boldsymbol{T}_{I B}\left[\begin{array}{lll}
u & v & w
\end{array}\right]^{\mathrm{T}}=\boldsymbol{T}_{B I}^{T}\left[\begin{array}{lll}
u & v & w
\end{array}\right]^{\mathrm{T}}
$$

Where $x_{g}, y_{g}, z_{g}$ are the components of the position vector of the hypersonic vehicle with respect to the ground inertial frame system. 


\subsection{Engagement Dynamics in the 3D Space}

The strategy that makes the LOS angular rates converge to zero is adopted in this work to get signal from outer loop. The 3D relative dynamics ${ }^{[15]}$ between the target and vehicle can be denoted as follows:

$$
\left\{\begin{array}{l}
r=\sqrt{x_{r}^{2}+y_{r}^{2}+z_{r}^{2}} \\
\lambda_{A}=\arctan \left(z_{r} / x_{r}\right) \\
\lambda_{E}=\arctan y_{r} / \sqrt{x_{r}^{2}+z_{r}^{2}} \\
\dot{r}=\dot{x}_{r} \cos \lambda_{E} \cos \lambda_{A}+\dot{z}_{r} \cos \lambda_{E} \sin \lambda_{A}+\dot{y}_{r} \sin \lambda_{E} \\
\dot{\lambda}_{A}=\left(\dot{z}_{r} \cos \lambda_{A}-\dot{x}_{r} \sin \lambda_{A}\right) / r \cos \lambda_{E} \\
\dot{\lambda}_{E}=\left(\dot{y}_{r} \cos \lambda_{E}-\dot{z}_{r} \sin \lambda_{E} \sin \lambda_{A}-\dot{x}_{r} \sin \lambda_{E} \cos \lambda_{A}\right) / r
\end{array}\right.
$$

Where: $x_{r}=x_{t}-x_{g}, y_{r}=y_{t}-y_{g}, z_{r}=z_{t}-z_{g}, \dot{x}_{r}=\dot{x}_{t}-\dot{x}_{g}, \dot{y}_{r}=\dot{y}_{t}-\dot{y}_{g}, \dot{z}_{r}=\dot{z}_{t}-\dot{z}_{g}$, and $x_{t}, y_{t}, z_{t}$ are the components of the position vector of the target in ground inertial frame system., $r$ is the relative distance between the vehicle and target, $\lambda_{A}, \lambda_{E}$ are the azimuth and elevation angles of the line of sight, respectively.

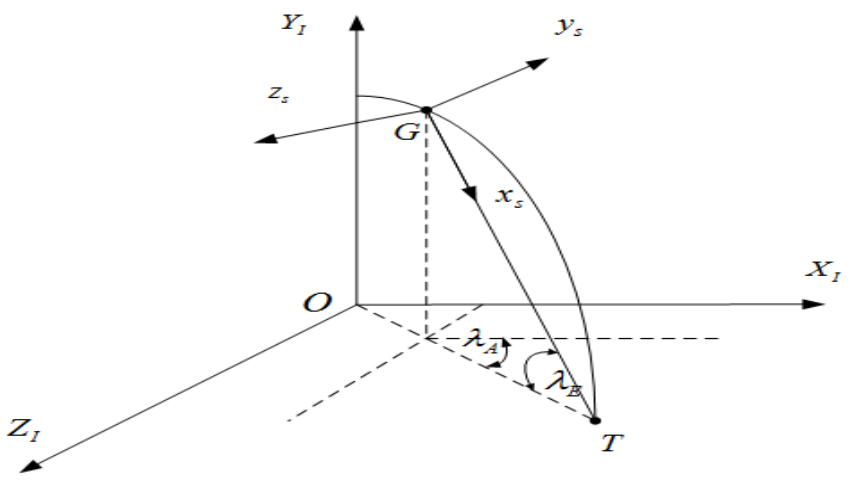

Figure 1 Geometry of the relationship between vehicle and target

\section{Loops design}

Figure 2 shows the structure of the partial integrated guidance and control method which adopted in this work.

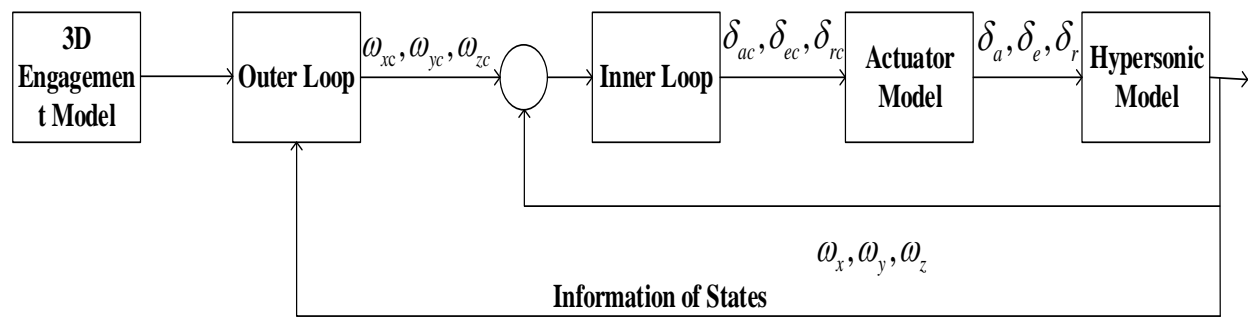

Figure 2. Structure relationship between the outer and inner loop

\subsection{Outer Loop Design}

Differentiate Eq.(3) with respect to time once:

$$
\left[\begin{array}{lll}
\ddot{x}_{g} & \ddot{y}_{g} & \ddot{z}_{g}
\end{array}\right]^{\mathrm{T}}=2 \boldsymbol{T}_{2}\left[\begin{array}{llll}
\dot{q}_{0} & \dot{q}_{1} & \dot{q}_{2} & \dot{q}_{3}
\end{array}\right]^{\mathrm{T}}+\boldsymbol{T}_{I B}\left[\begin{array}{lll}
\dot{u} & \dot{v} & \dot{w}
\end{array}\right]^{\mathrm{T}}
$$

Where:

$$
\boldsymbol{T}_{2}=\left[\begin{array}{cccc}
q_{0} u-q_{3} v+q_{2} w & q_{1} u+q_{2} v+q_{3} w & -q_{2} u+q_{1} v+q_{0} w & -q_{3} u-q_{0} v+q_{1} w \\
q_{3} u+q_{0} v-q_{1} w & q_{2} u-q_{1} v-q_{0} w & q_{1} u+q_{2} v+q_{3} w & q_{0} u-q_{3} v+q_{2} w \\
-q_{2} u+q_{1} v+q_{0} w & q_{3} u+q_{0} v-q_{1} w & -q_{0} u+q_{3} v-q_{2} w & q_{1} u+q_{2} v+q_{3} w
\end{array}\right]
$$

Substituting Eq.(2) into Eq.(5):

$$
\boldsymbol{T}_{2} \boldsymbol{T}_{1}\left[\begin{array}{llll}
\dot{q}_{0} & \dot{q}_{1} & \dot{q}_{2} & \dot{q}_{3}
\end{array}\right]^{\mathrm{T}}+\boldsymbol{T}_{I B}\left[\begin{array}{ccc}
\dot{u} & \dot{v} & \dot{w}
\end{array}\right]^{\mathrm{T}} \triangleq \boldsymbol{T}_{3}\left[\begin{array}{lll}
\omega_{x} & \omega_{y} & \omega_{z}
\end{array}\right]^{\mathrm{T}}+\boldsymbol{T}_{I B}\left[\begin{array}{lll}
\dot{u} & \dot{v} & \dot{w}
\end{array}\right]^{\mathrm{T}}
$$


Where:

From Eq.(4) we have:

$$
\begin{aligned}
& \boldsymbol{T}_{3}=\left[\begin{array}{lll}
m_{11} & m_{12} & m_{13} \\
m_{21} & m_{22} & m_{23} \\
m_{31} & m_{32} & m_{33}
\end{array}\right] \\
& m_{11}=2\left(q_{0} q_{3}-q_{1} q_{2}\right) v+2\left(q_{0} q_{2}+q_{1} q_{3}\right) w \\
& m_{12}=-2\left(q_{0} q_{2}+q_{1} q_{3}\right) u-\left(q_{0}^{2}+q_{1}^{2}-q_{2}^{2}-q_{3}^{2}\right) w \\
& m_{13}=2\left(q_{1} q_{2}-q_{0} q_{3}\right) u-\left(q_{0}^{2}+q_{1}^{2}-q_{2}^{2}-q_{3}^{2}\right) v \\
& m_{21}=2\left(q_{2} q_{3}-q_{0} q_{1}\right) v-\left(q_{0}^{2}-q_{1}^{2}+q_{2}^{2}-q_{3}^{2}\right) w \\
& m_{22}=2\left(q_{0} q_{1}-q_{2} q_{3}\right) u+2\left(q_{0} q_{3}+q_{1} q_{2}\right) w \\
& m_{23}=\left(q_{0}^{2}-q_{1}^{2}+q_{2}^{2}-q_{3}^{2}\right) u-2\left(q_{0} q_{3}+q_{1} q_{2}\right) w \\
& m_{31}=\left(q_{0}^{2}-q_{1}^{2}-q_{2}^{2}+q_{3}^{2}\right) v-2\left(q_{0} q_{1}-q_{2} q_{3}\right) w \\
& m_{32}=\left(-q_{0}^{2}+q_{1}^{2}+q_{2}^{2}-q_{3}^{2}\right) u+2\left(q_{1} q_{3}-q_{0} q_{2}\right) w \\
& m_{33}=2\left(q_{0} q_{1}+q_{2} q_{3}\right) u+2\left(q_{0} q_{2}-q_{1} q_{3}\right) v
\end{aligned}
$$

$$
\left[\begin{array}{ll}
\ddot{\lambda}_{A} & \ddot{\lambda}_{E}
\end{array}\right]^{\mathrm{T}}=\boldsymbol{T}_{4}+\boldsymbol{T}_{5}\left[\ddot{x}_{t}-\ddot{x}_{g} \quad \ddot{y}_{t}-\ddot{y}_{g} \quad \ddot{z}_{t}-\ddot{z}_{g}\right]^{\mathrm{T}}
$$

Where:

$$
\begin{gathered}
\boldsymbol{T}_{4}=\left[\begin{array}{ccc}
2 \dot{\lambda}_{A} \dot{\lambda}_{E} \tan \lambda_{E}-2 \dot{r}_{A} / r & -\lambda_{A}^{2} \sin \lambda_{E} \cos \lambda_{E}-2 \dot{r} \dot{\lambda}_{E} / r
\end{array}\right]^{\mathrm{T}} \\
\boldsymbol{T}_{5}=\left[\begin{array}{ccc}
-\sin \lambda_{A} / r \cos \lambda_{E} & 0 & -\cos \lambda_{A} / r \cos \lambda_{E} \\
-\sin \lambda_{E} \cos \lambda_{A} / r & \cos \lambda_{E} / r & \sin \lambda_{E} \sin \lambda_{A} / r
\end{array}\right]
\end{gathered}
$$

To make the LOS angle converge to zero, the sliding mode surface is designed as follow:

$$
s_{1}=\left[\begin{array}{ll}
s_{11} & s_{21}
\end{array}\right]^{\mathrm{T}}=\left[\begin{array}{ll}
\dot{\lambda}_{A} & \dot{\lambda}_{E}
\end{array}\right]^{\mathrm{T}}
$$

And adopt the following terminal sliding mode reaching law:

$$
\dot{\boldsymbol{s}}_{1 d}=-\boldsymbol{k}_{11} \boldsymbol{s}_{1}-\boldsymbol{k}_{12}\left[\left|s_{11}\right|^{d_{1}} \operatorname{sgn}\left(s_{11}\right) \quad\left|s_{12}\right|^{d_{2}} \operatorname{sgn}\left(s_{12}\right)\right]^{\mathrm{T}}
$$

Where, $\boldsymbol{k}_{11}, \boldsymbol{k}_{12} \in \boldsymbol{R}^{2 \times 2}, 0<d_{1}, d_{2}<1$ need to be designed manually. Differentiate Eq. (8) with respect to time once:

$$
\dot{s}_{1}=\left[\begin{array}{ll}
\dot{s}_{11} & \dot{s}_{21}
\end{array}\right]^{\mathrm{T}}=\left[\begin{array}{ll}
\ddot{\lambda}_{A} & \ddot{\lambda}_{E}
\end{array}\right]^{\mathrm{T}}=\boldsymbol{T}_{4}+\boldsymbol{T}_{5}\left[\begin{array}{lll}
\ddot{x}_{t} & \ddot{y}_{t} & \ddot{z}_{t}
\end{array}\right]^{\mathrm{T}}-\boldsymbol{T}_{5}\left[\begin{array}{lll}
\ddot{x}_{g} & \ddot{y}_{g} & \ddot{z}_{g}
\end{array}\right]^{\mathrm{T}}
$$

Substituting Eq. (6) into equation above:

$$
\dot{\boldsymbol{s}}_{1}=\boldsymbol{T}_{4}+\boldsymbol{T}_{5}\left[\begin{array}{lll}
\ddot{x}_{t} & \ddot{y}_{t} & \ddot{z}_{t}
\end{array}\right]^{\mathrm{T}}-\boldsymbol{T}_{5} \boldsymbol{T}_{3}\left[\begin{array}{lll}
\omega_{x} & \omega_{y} & \omega_{z}
\end{array}\right]^{\mathrm{T}}-\boldsymbol{T}_{5} \boldsymbol{T}_{I B}\left[\begin{array}{lll}
\dot{u} & \dot{v} & \dot{w}
\end{array}\right]^{\mathrm{T}} \triangleq \boldsymbol{A}-\boldsymbol{B}\left[\begin{array}{ccc}
\omega_{x} & \omega_{y} & \omega_{z}
\end{array}\right]^{\mathrm{T}}
$$

Where:

$\boldsymbol{A}=\boldsymbol{T}_{4}-\boldsymbol{T}_{5} \boldsymbol{T}_{I B}\left[\begin{array}{lll}\dot{u} & \dot{v} & \dot{w}\end{array}\right]^{\mathrm{T}}, \boldsymbol{B}=\boldsymbol{T}_{5} \boldsymbol{T}_{3}$, This work aims to the fixed target, so $\ddot{x}_{t}=\ddot{y}_{t}=\ddot{z}_{t}=0$. Combine Eq.(9) we get:

$$
\left[\begin{array}{lll}
\omega_{x c} & \omega_{y c} & \omega_{z c}
\end{array}\right]^{\mathrm{T}}=\boldsymbol{B}^{-1}\left(\boldsymbol{A}-\dot{\boldsymbol{s}}_{1 d}\right)
$$

We can get the desired body rates signal which guide the vehicle to the target from equation given above.

\subsection{Inner Loop Design}

Eq.(1) can be rewritten as

$$
\left[\begin{array}{lll}
\dot{\omega}_{x} & \dot{\omega}_{y} & \dot{\omega}_{z}
\end{array}\right]^{\mathrm{T}}=\left[\begin{array}{lll}
\Delta l & \Delta n & \Delta m
\end{array}\right]^{\mathrm{T}}+\boldsymbol{E}\left[\begin{array}{lll}
\delta_{a} & \delta_{e} & \delta_{r}
\end{array}\right]^{\mathrm{T}}
$$

Where: 


$$
\boldsymbol{E}=\left[\begin{array}{ccc}
K_{x} & 0 & 0 \\
0 & K_{y} & 0 \\
0 & 0 & K_{z}
\end{array}\right]\left[\begin{array}{lll}
C_{l, 1} & C_{l, 2} & C_{l, 3} \\
C_{n, 1} & C_{n, 2} & C_{n, 3} \\
C_{m, 1} & C_{m, 2} & C_{m, 3}
\end{array}\right]
$$

$K_{x}=\frac{q S a}{I_{x x}}, K_{y}=\frac{q S a}{I_{y y}}, K_{z}=\frac{q S c}{I_{z z}}, C_{i, j}, i=l, n, m, j=1,2,3$ are the coefficients respect to the first power of fins deflection of the left elevon, right elevon and the rudder.

$$
\begin{aligned}
& \Delta l=\left(I_{y y}-I_{z z}\right) \omega_{y} \omega_{z} / I_{x x}+K_{x} \Delta C_{l} \\
& \Delta n=\left(I_{z z}-I_{x x}\right) \omega_{z} \omega_{x} / I_{x x}+K_{y} \Delta C_{n} \\
& \Delta m=\left(I_{x x}-I_{y y}\right) \omega_{x} \omega_{y} / I_{x x}+K_{z} \Delta C_{m}
\end{aligned}
$$

Where, $\Delta C_{l}, \Delta C_{n}, \Delta C_{m}$ are the remaining terms in the aerodynamic moment coefficients.

To track the desire body rates signal generated by outer loop, we design the following sliding mode surface:

$$
\boldsymbol{s}_{2}=\left[\begin{array}{lll}
s_{21} & s_{22} & s_{23}
\end{array}\right]^{\mathrm{T}}=\left[\begin{array}{lll}
\omega_{x}-\omega_{x c} & \omega_{y}-\omega_{y c} & \omega_{z}-\omega_{z c}
\end{array}\right]^{\mathrm{T}}
$$

And adopt the reaching law as follow:

$$
\dot{\boldsymbol{s}}_{2 d}=-\boldsymbol{k}_{21} \boldsymbol{s}_{2}-\boldsymbol{k}_{22}\left[\operatorname{sgn}\left(s_{21}\right) \operatorname{sgn}\left(s_{22}\right) \quad \operatorname{sgn}\left(s_{23}\right)\right]^{\mathrm{T}}
$$

Where, $k_{21}, k_{22} \in R^{3 \times 3}$ are the design parameters. Combining Eq.(10) we can obtain the desired fin flection command:

$$
\left[\begin{array}{lll}
\delta_{a c} & \delta_{e c} & \delta_{r c}
\end{array}\right]^{\mathrm{T}}=\boldsymbol{E}^{-1}\left(\dot{\boldsymbol{s}}_{2 d}+\left[\begin{array}{lll}
\dot{\omega}_{x c} & \dot{\omega}_{y c} & \dot{\omega}_{z c}
\end{array}\right]^{\mathrm{T}}-\left[\begin{array}{lll}
\Delta l & \Delta n & \Delta m
\end{array}\right]^{\mathrm{T}}\right)
$$

Considering the response delay of the fin actuators, the actual left elevon, right elevon, and rudder deflections $\delta_{a c}, \delta_{e c}, \delta_{r c}$ are treated as the inputs of the second-order fin actuators that yield the actual fin deflections $\delta_{a}, \delta_{e}, \delta r$ applied to hypersonic vehicles.

$$
\ddot{\delta}_{i}+2 \xi \omega_{n} \dot{\delta}_{i}+\omega_{n}^{2} \delta_{i}=\omega_{n}^{2} \delta_{i c}, i=a, e, r
$$

Where, $\omega_{n}=20 \mathrm{~Hz}$ the natural frequency of the fin actuator, $\xi=0.7$ is the damping ratio .the domain of the fin flection is $\left(-20^{\circ}, 20^{\circ}\right)$, and the maximum fin deflection rate is $100^{\circ} / \mathrm{s}$.

\section{Simulation}

\subsection{Simulation Conditions and Parameters}

All the initial conditions and the design parameters are listed in table 1.

Table 1. Initial value of the flight

\begin{tabular}{|c|c|c|c|c|}
\hline & $x_{g 0} / m$ & $y_{g 0} / m$ & $z_{g 0} / m$ & $\varphi_{0} /^{\circ}$ \\
\hline case1 & 4000 & 22500 & 2000 & 5 \\
\hline case2 & 0 & 25000 & 0 & 5 \\
\hline & $\psi_{0} l^{\circ}$ & $\gamma_{0} /^{\circ}$ & $V_{0} / m \cdot s^{-1}$ & $\alpha_{0} /{ }^{\circ}$ \\
\hline case1 & 2 & -5 & 2400 & 3 \\
\hline case2 & -5 & -10 & 2500 & 0 \\
\hline & $\beta_{0} l^{\circ}$ & $\omega_{x 0} /{ }^{\circ} \cdot s^{-1}$ & $\omega_{y 0} /{ }^{\circ} \cdot s^{-1}$ & $\omega_{z 0} / s^{-1}$ \\
\hline case1 & -2 & -6 & 6 & 4 \\
\hline case2 & 0 & 5 & 5 & 3 \\
\hline
\end{tabular}

The initial value of the quaternion $\left[\begin{array}{llll}q_{00} & q_{10} & q_{20} & q_{30}\end{array}\right]^{T}$ can get from the following equation, where $\varphi_{0}, \psi_{0}, \gamma_{0}$ are the initial pitch, yaw and roll angle. 


$$
\left[\begin{array}{llll}
q_{00} & q_{10} & q_{20} & q_{30}
\end{array}\right]^{\mathrm{T}}=\left[\begin{array}{l}
\cos \frac{\varphi_{0}}{2} \cos \frac{\psi_{0}}{2} \cos \frac{\gamma_{0}}{2}-\cos \frac{\varphi_{0}}{2} \cos \frac{\psi_{0}}{2} \cos \frac{\gamma_{0}}{2} \\
\cos \frac{\varphi_{0}}{2} \cos \frac{\psi_{0}}{2} \sin \frac{\gamma_{0}}{2}+\sin \frac{\varphi_{0}}{2} \sin \frac{\psi_{0}}{2} \cos \frac{\gamma_{0}}{2} \\
\cos \frac{\varphi_{0}}{2} \sin \frac{\psi_{0}}{2} \cos \frac{\gamma_{0}}{2}+\sin \frac{\varphi_{0}}{2} \cos \frac{\psi_{0}}{2} \sin \frac{\gamma_{0}}{2} \\
\sin \frac{\varphi_{0}}{2} \cos \frac{\psi_{0}}{2} \cos \frac{\gamma_{0}}{2}-\cos \frac{\varphi_{0}}{2} \sin \frac{\psi_{0}}{2} \sin \frac{\gamma_{0}}{2}
\end{array}\right]
$$

The initial velocity components of the vehicle $\left[\begin{array}{lll}u_{0} & v_{0} & w_{0}\end{array}\right]^{\mathrm{T}}$ are given by the following equation: $\left[\begin{array}{lll}u_{0} & v_{0} & w_{0}\end{array}\right]^{\mathrm{T}}=\boldsymbol{T}_{B V}\left[\begin{array}{lll}V_{0} & 0 & 0\end{array}\right]^{\mathrm{T}}$, where:

$$
\boldsymbol{T}_{B V}=\left[\begin{array}{ccc}
\cos \alpha_{0} \cos \beta_{0} & \sin \alpha_{0} & -\cos \alpha_{0} \sin \beta_{0} \\
-\sin \alpha_{0} \cos \beta_{0} & \cos \alpha_{0} & \sin \alpha_{0} \sin \beta_{0} \\
\sin \beta_{0} & 0 & \cos \beta_{0}
\end{array}\right]
$$

Set the target position as follow: $x_{t}=150000 m, y_{t}=0 m, z_{t}=30000 m$; the design parameters in this paper are given by: $d_{1}=d_{2}=0.6, \boldsymbol{k}_{11}=\operatorname{diag}(0.015,0.02), \boldsymbol{k}_{12}=\operatorname{diag}(0.005,0.005), \boldsymbol{k}_{21}=\operatorname{diag}(1.5,1.5)$, $\boldsymbol{k}_{22}=\operatorname{diag}(0.0015,0.0015)$.

\subsection{Simulation results}

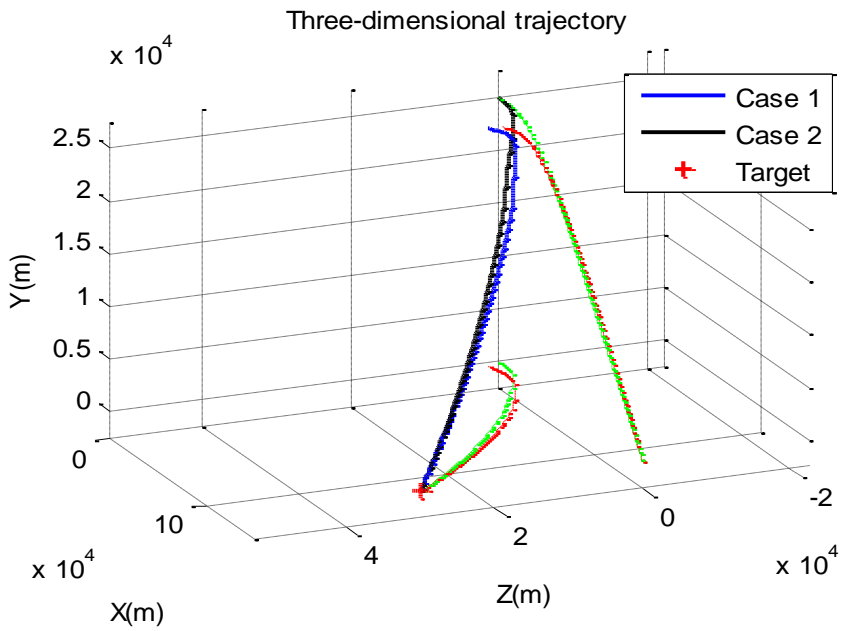

Figure 3 Trajectories of the vehicle in 3D space

In Fig. 3 the red and green lines represent the projection of the two dimensional trajectory of hypersonic vehicle on XOY and XOZ surfaces respectively.

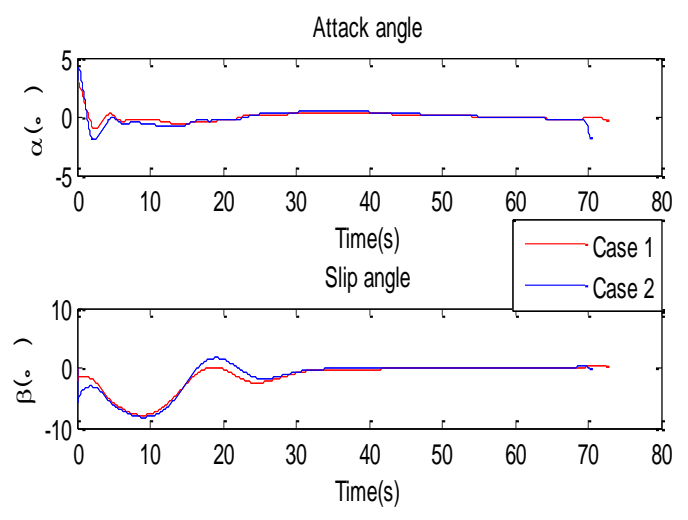

Figure 4. Curves of the angle of attack and sideslip.
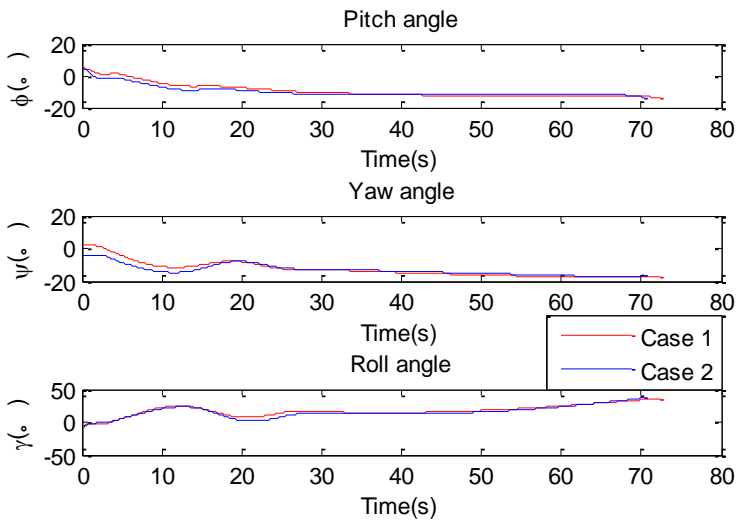

Figure 5. Curves of the body angles. 


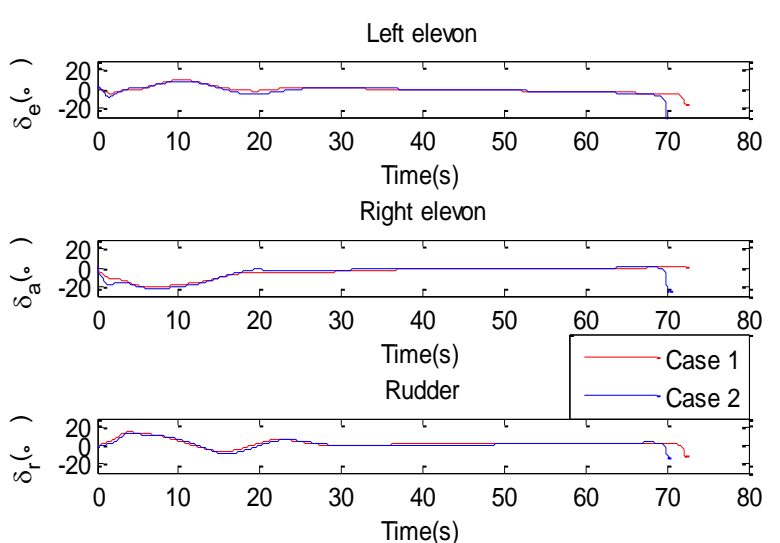

Figure 6. Curves of the fin deflections.

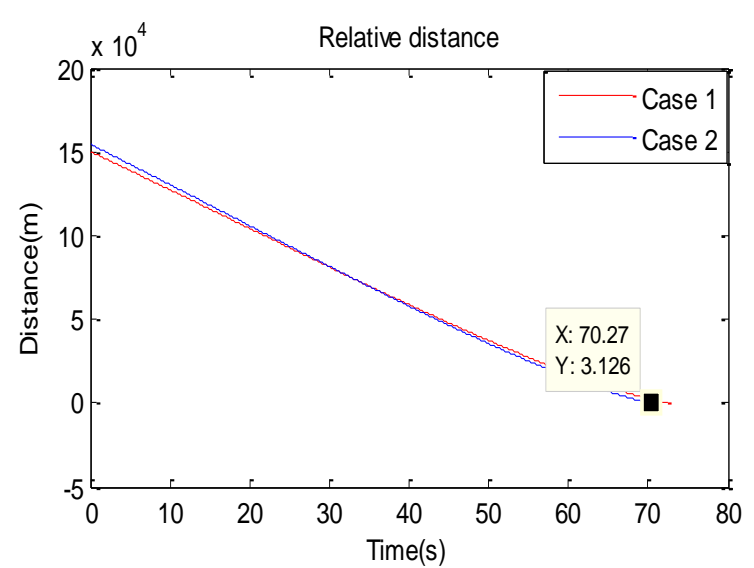

Figure 7. Curves of the relative distance.

Fig.4-6 give the simulation results, and we can find the states of the vehicle are steady, and the fin deflections are small and within the limitation of the actuator.

This paper gives different initial conditions, through the simulation we can see the hypersonic vehicle to the accuracy of the 3.484m hit the target in Fig.7, and the same GHV model and using the IGC method based on literature [16], the accuracy of $6.562 \mathrm{~m}$, using this method can improve the accuracy, and the state of aircraft and the rudder angle changes stable during the flight.

\section{Conclusion}

In order to solve the guidance and control problem of hypersonic vehicle, the model of six degrees of freedom of hypersonic vehicle using quaternion to avoid the instable problem in maneuver flight is established .And this paper uses full information of the relative motion, PIGC method is adopted to make the whole flight stable and reach a high accuracy.

\section{References}

[1] Sun C Y, Mu C X and Yu Y 2013 ACTA Automatica Sinica. 39 1901-13.

[2] Li H F 2012 Guidance and control technology of hypersonic vehicles (Beijing: China Astronautic Publishing House)

[3] Zhu J W, Liu L H and Tang G J 2015 Journal of Aero-space Engineering. 229 2321-35.

[4] Zhu J W, Liu L H and Tang G J2014 Journal of national university of defense technology. 36 24-29.

[5] Song C, Zhao G R and Gai J F 2012 Systems Engineering and Electronics. 34 1231-34.

[6] Zeng X F,Wang X H and Zhang Jing 2012 Journal of Beijing University of Aeronautics and Astronautics. 38 1454-58.

[7] $\mathrm{Hu} X, \mathrm{Wu} \mathrm{L}$ and $\mathrm{Hu} \mathrm{C} 2012$ Iet Control Theory \& Applications. 6 1238-49.

[8] Zhang T, Zhou J and Guo J 2014 ACTA Aeronautica ET Astronautica Sinica 35 215-22.

[9] Xu B , Shi Z K, Yang C G and Wang S X 2013 Nonlinear Dynamics, 73 1849-61.

[10] Zhao S Y 1992 Homing guidance and control system design for air defence missiles (Beijing: China Astronautics Publishing House) pp 136-140.

[11] Keshmiri S, Mirmirani M and Colgren R. 2004 Six-DOF Modelling and Simulation of a Generic Hypersonic Vehicle for Conceptual Design Studies (Rhode Island: AIAA Modelling and Simulation Technologies Conference and Exhibit) pp 16-19.

[12] Wu S T, Fei Y 2006 Flight control system (Beijing: Beihang University Press) pp 262-275.

[13] Zhao H Y 1997 Spacecraft reentry dynamics and guidance (Changsha: Press of National University of Defence Technology) pp 56-64.

[14] Qin L, Zhang W D and Pan F 2006 Journal of North University of China 27 276-9.

[15] Zhou D 2002 New Guidance Laws for Homing Missile (Beijing: National Defence Industry Press) 
[16] Wang J H, Liu L H, Wang P and Tang G J 2017 ACTA Aeronautica ET Astronautica Sinica 38 207-19.

\begin{tabular}{|c|c|c|c|c|}
\hline First name & Surname & Institution & Email address & Title \\
\hline Qingkai & Liu & $\begin{array}{c}\text { Xi'an Research Institute of High-Tech, } \\
\text { Xi'an, China }\end{array}$ & lqk_rkue@ foxmail.com & student \\
\hline Jian & Chen & $\begin{array}{c}\text { Xi'an Research Institute of High-Tech, } \\
\text { Xi'an, China }\end{array}$ & chenjian_rk@126.com & Prof \\
\hline Lixin & Wang & $\begin{array}{c}\text { Xi'an Research Institute of High-Tech, } \\
\text { Xi'an, China }\end{array}$ & wanglixin_rk@126.com & Prof \\
\hline
\end{tabular}

